\title{
Cracked orthotropic strip with clamped boundaries
}

By H. G. Georgiadis*) and G. A. Papadopoulos**), Dept. Mech. Engng. and Appl. Mech., The University of Michigan, 2250 G. G. Brown Bldg, Ann Arbor, Mich. 48109-2125, USA

\section{Introduction}

One of the basic aims of the linear elastic fracture mechanics (LEFM) in recent years is the determination of stress intensity factors in complicated situations regarding geometric features and/or mechanical behavior [1]. This reflects the general claim for fracture mechanics solutions to represent engineering reality instead of idealized situations. Of course, mathematical complexities set some bounds up to this policy and then one has to resort to numerical methods. However, there are still many problems in the realm of $L E F M$ which admit an analytical treatment. One of these is the problem solved here.

This concerns a long strip made by orthotropic material containing a long crack. The case of clamped strip boundaries will be discussed here. The alternative way of loading, i.e. the shear free strip boundaries, has been considered in a recent paper of ours [2]. The present work follows the analysis therein closely.

Knauss [3] and Rice [4] have solved the respective isotropic problem, whereas Nilsson [5] and Popelar et al. [6] considered dynamic crack motion in an elastic and viscoelastic strip. Relative to the present work are the ones in [7-11] where, however, different geometric features were encountered.

The solution here was accomplished by Fourier transforms and the Wiener-Hopf technique. The procedure will be described briefly.

\section{Governing equations}

Consider a linear elastic orthotropic body in the form depicted in Fig. 1. Then, with respect to the principal material-axes, the elastic constitutive expression relating the in-plane stresses and displacements is [12]

$$
\left[\begin{array}{c}
\sigma_{x} \\
\sigma_{y} \\
\tau_{x y}
\end{array}\right]=\left[\begin{array}{ccc}
c_{11} & c_{12} & 0 \\
c_{12} & c_{22} & 0 \\
0 & 0 & c_{66}
\end{array}\right]\left[\begin{array}{c}
\varepsilon_{x} \\
\varepsilon_{y} \\
\gamma_{x y}
\end{array}\right]
$$

where the components of the stiffness matrix are given in terms of the engineering material constants as follows

$$
c_{11}=E_{1} /\left(1-v_{1} v_{2}\right), \quad c_{12}=v_{2} E_{1} /\left(1-v_{1} v_{2}\right), \quad c_{22}=E_{2} /\left(1-v_{1} v_{2}\right), \quad c_{66}=G .
$$

*) Permanent address: 33-35 G. Papandreou St., 16231 Athens, Greece.

**) Mechanics Division, The National Technical University of Athens, Greece. 
Figure 1

A long orthotropic strip with clamped boundaries containing a crack opened by vertical displacements.

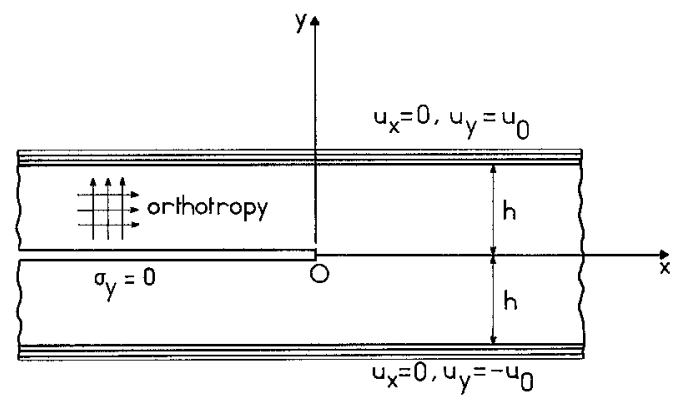

In the above relations, the subscripts 1 and 2 refer to the principal directions of material symmetry which coincide here with the $x$ and $y$ reference axes. Only four elastic constants are independent, the fifth given by

$$
v_{2} E_{1}=v_{1} E_{2} \text {. }
$$

Following Ref. [2], we introduce the $\phi$ - and $\psi$-displacement potential by the relations

$$
u_{x}=\frac{\partial}{\partial x}(\phi+\psi), \quad u_{y}=\frac{\partial}{\partial y}(\alpha \phi+b \psi), \quad u_{z}=0
$$

where

$$
\alpha=\frac{c_{11} \beta_{1}-c_{66}}{c_{12}+c_{66}}=\frac{\left(c_{12}+c_{66}\right) \beta_{1}}{c_{22}-c_{66} \beta_{1}}, \quad b=\frac{c_{11} \beta_{2}-c_{66}}{c_{12}+c_{66}}=\frac{\left(c_{12}+c_{66}\right) \beta_{2}}{c_{22}-c_{66} \beta_{2}}
$$

and $\beta_{1}, \beta_{2}$ the roots of the characteristic equation

$$
c_{11} c_{66} \beta^{2}+\left(c_{12}^{2}+2 c_{12} c_{66}-c_{11} c_{22}\right) \beta+c_{22} c_{66}=0 \text {. }
$$

It can be shown further that the displacement potentials satisfy the following Laplace type differential equations

$$
\frac{\partial^{2} \phi}{\partial x^{2}}+\beta_{1} \frac{\partial^{2} \phi}{\partial y^{2}}=0, \quad \frac{\partial^{2} \psi}{\partial x^{2}}+\beta_{2} \frac{\partial^{2} \psi}{\partial y^{2}}=0
$$

and the stresses are given in terms of $\phi$ and $\psi$ as

$$
\begin{aligned}
& \sigma_{x}=\frac{(1+\alpha) c_{66}}{\beta_{1}} \frac{\partial^{2} \phi}{\partial y^{2}}+\frac{(1+b) c_{66}}{\beta_{2}} \frac{\partial^{2} \psi}{\partial y^{2}} \\
& \sigma_{y}=-(1+\alpha) c_{66} \frac{\partial^{2} \phi}{\partial x^{2}}-(1+b) c_{66} \frac{\partial^{2} \psi}{\partial x^{2}} \\
& \tau_{x y}=c_{66}\left[(1+\alpha) \frac{\partial^{2} \phi}{\partial x \partial y}+(1+b) \frac{\partial^{2} \psi}{\partial x \partial y}\right] .
\end{aligned}
$$

\section{Analysis}

The semi-infinite crack is opened by constant vertical displacements $u_{0}$ applied to the strip boundaries, whereas these boundaries are clamped in the $x$-direction. According to Popelar and Atkinson [6] this type of loading is more convenient for fracture studies 
than the shear-free boundaries. To apply the Wiener-Hopf technique the following auxiliary problem is considered

$$
\begin{array}{lll}
u_{x}(x, h)=0 & \text { for } & -\infty<x<\infty \\
u_{y}(x, h)=0 & \text { for } & -\infty<x<\infty \\
\tau_{x y}(x, 0)=0 & \text { for } & -\infty<x<\infty \\
\sigma_{y}(x, 0)=\sigma_{0} & \text { for } & -\infty<x<0 \\
u_{y}(x, 0)=0 & \text { for } & 0<x<\infty
\end{array}
$$

Then, by a trivial superposition we may arrive again at the original problem. The proper value of $\sigma_{0}$ in the above boundary conditions and for plane-stress may be derived by Eq. (1) as

$$
\sigma_{0}=-\left(c_{12} v_{2}+c_{22}\right) \frac{u_{0}}{h}
$$

Obviously, such a superposition does not affect the value of the stress intensity factor obtained by solving problem (7).

Now we introduce two as yet unknown functions the determination of which completes the solution of problem (7):

$$
\begin{aligned}
& \sigma_{y}(x, 0)=m(x) \quad \text { for } \quad 0<x<\infty \\
& u_{y}(x, 0)=n(x) \quad \text { for } \quad-\infty<x<0 .
\end{aligned}
$$

Applying the Fourier transform Eqs. (5) become [13]

$$
\begin{aligned}
& -\omega^{2} \phi^{*}(\omega, y)+\beta_{1} \frac{\partial^{2}}{\partial y^{2}} \phi^{*}(\omega, y)=0 \\
& -\omega^{2} \psi^{*}(\omega, y)+\beta_{2} \frac{\partial^{2}}{\partial y^{2}} \psi^{*}(\omega, y)=0
\end{aligned}
$$

which have general solutions of the form $\left(\gamma_{j}=\beta_{j}^{-1 / 2}(j=1,2)\right)$

$$
\begin{aligned}
& \phi^{*}(\omega, y)=A(\omega) e^{y_{1} \omega y}+B(\omega) e^{-\gamma_{1} \omega y} \\
& \psi^{*}(\omega, y)=C(\omega) e^{y_{2} \omega y}+D(\omega) e^{-\gamma_{2} \omega y} .
\end{aligned}
$$

The transforms of the functions of interest are defined as

$$
\begin{aligned}
& m_{+}^{*}(\omega)=(2 \pi)^{-1 / 2} \int_{0}^{\infty} m(x) e^{i \omega x} \mathrm{~d} x \\
& n_{-}^{*}(\omega)=(2 \pi)^{-1 / 2} \int_{-\infty}^{0} n(x) e^{i \omega x} \mathrm{~d} x .
\end{aligned}
$$

Then, application of the Fourier transform to the boundary conditions in conjunction with the transformed Eqs. (6) results in an algebraic system of five equations with the six unknown functions $A(\omega), B(\omega), C(\omega), D(\omega), m_{+}(\omega)$ and $n_{-}(\omega)$. Some tedious algebra eliminates $A(\omega), \ldots, D(\omega)$ and reduces the system to the following Wiener-Hopf equation

$$
m_{+}^{*}(\omega)=-\frac{c_{66}}{\gamma_{1} \gamma_{2}(\alpha-b)} \quad K(\omega) n_{-}^{*}(\omega)-\frac{\sigma_{0}}{i(2 \pi)^{1 / 2} \omega}
$$


where the Kernel $K(\omega)=\omega F(\omega)[G(\omega)]^{-1}$ is given by

$$
\begin{aligned}
F(\omega)= & (1+\alpha)\left\{2 \gamma_{1} \gamma_{2}(1+\alpha) b-(1+b) \gamma_{2}\left[\left(b \gamma_{2}+\alpha \gamma_{1}\right) \cosh \left(\gamma_{1} h \omega-\gamma_{2} h \omega\right)\right.\right. \\
& \left.\left.-\left(b \gamma_{2}-\alpha \gamma_{1}\right) \cosh \left(\gamma_{1} h \omega+\gamma_{2} h \omega\right)\right]\right\}+(1+b)\left\{2 \gamma_{1} \gamma_{2}(1+b) \alpha\right. \\
& -(1+\alpha) \gamma_{1}\left[\left(b \gamma_{2}+\alpha \gamma_{1}\right) \cosh \left(\gamma_{1} h \omega-\gamma_{2} h \omega\right)+\left(b \gamma_{2}-\alpha \gamma_{1}\right)\right. \\
& \left.\left.\cdot \cosh \left(\gamma_{1} h \omega+\gamma_{2} h \omega\right)\right]\right\}, \\
G(\omega)= & \left(b \gamma_{2}-\alpha \gamma_{1}\right) \sinh \left(\gamma_{1} h \omega+\gamma_{2} h \omega\right) \\
& -\left(b \gamma_{2}+\alpha \gamma_{1}\right) \sinh \left(\gamma_{1} h \omega-\gamma_{2} h \omega\right) .
\end{aligned}
$$

The Kernel takes the following asymptotic forms for small and large $\omega$ 's

$$
\begin{aligned}
& \lim _{\omega \rightarrow 0} K(\omega)=\frac{\gamma_{1} \gamma_{2} \cdot(\alpha-b)^{2}}{\left(\alpha \gamma_{1}^{2}-b \gamma_{2}^{2}\right) h} \\
& \lim _{\omega \rightarrow \infty} \frac{K(\omega)}{\omega}=(1+\alpha)(1+b)\left(\gamma_{2}-\gamma_{1}\right) .
\end{aligned}
$$

Following now the classical Wiener-Hopf method [14] and the procedure in Refs. $[2,5,15]$ we may find

$$
m_{+}^{*}(\omega)=-\frac{\sigma_{0} K_{+}(\omega)}{i(2 \pi)^{1 / 2} \omega}\left[\frac{1}{K_{+}(\omega)}-\frac{1}{K_{+}(0)}\right]
$$

and further

$$
\lim _{\omega \rightarrow \infty} m_{+}^{*}(\omega)=-\frac{\sigma_{0}}{i(2 \pi)^{1 / 2} \omega}+\frac{\sigma_{0}}{i(2 \pi)^{1 / 2} \omega^{1 / 2} K_{+}(0)} \lim _{\omega \rightarrow \infty} \frac{K_{+}(\omega)}{\omega^{1 / 2}}
$$

or [13]

$$
\lim _{x \rightarrow 0^{+}} m(x)=-\frac{\sigma_{0}}{\pi^{1 / 2}}\left[\frac{\left(\gamma_{2}-\gamma_{1}\right)\left(\alpha \gamma_{1}^{2}-b \gamma_{2}^{2}\right)(1+\alpha)(1+b) h}{\gamma_{1} \gamma_{2}(a-b)^{2}}\right]^{1 / 2} x^{-1 / 2} .
$$

The above expression is the asymptotic form of the cleavage $\sigma_{y}(x, 0)-$ stress near the crack tip. Since the stress intensity factor is given by

$$
K_{I}=\lim _{x \rightarrow 0^{+}}\left[(2 \pi x)^{1 / 2} \cdot \sigma_{y}(x, 0)\right]
$$

we may easily find that in our case

$$
K_{I}=\left[\frac{2\left(\gamma_{2}-\gamma_{1}\right)\left(\alpha \gamma_{1}^{2}-b \gamma_{2}^{2}\right)(1+\alpha)(1+b)}{\gamma_{1} \gamma_{2}(\alpha-b)^{2}}\right]^{1 / 2} \frac{\left(c_{12} v_{2}+c_{22}\right) u_{0}}{h^{1 / 2}}
$$

\section{Results and discussion}

Obtaining the isotropic result of Rice [4] as a limiting case of our analysis seems to be cumbersome. This is due to the fact that both numerator and denominator in Eq. (21) tend to zero for $\alpha=b, \gamma_{1}=\gamma_{2}$, viz. for an isotropic material. Of course, application of L'Hospital rule may lead to an analytic result after some tedious algebra. However, in order to check our final result (21), we chose to work numerically. We consider the stress intensity factor for a nearly isotropic material with the following mechanical constants: 
$E_{1} / E_{2}=1.3 / 1.0, v_{1} / v_{2}=0.325 / 0.250=1.3, c_{66}=E_{2} / 2\left(1+v_{2}\right)=0.4$ (all values have been normalized in respect to $E_{2}$-modulus). In order to get the isotropic SIF, the values $E=E_{2}=1.0$ and $v=v_{2}=0.250$ were used.

In addition, we compare the results of (21) with those obtained by our formula for the shear-free type of boundary conditions in the same strip [2]. It is noticed that the latter case is reduced immediately to the isotropic one (see [5] for an analogy in elastodynamics).

The following stress intensity factor values were found in the three cases:

and

$$
K_{I}^{\text {iso }}=1.032 u_{0} h^{-1 / 2}, K_{I}^{\text {orth } 1}=1.145 u_{0} h^{-1 / 2} \text { (clamped boundaries) }
$$

$$
K_{I}^{\text {orth2 }}=1.105 u_{0} h^{-1 / 2} \text { (shear-free boundaries). }
$$

The above results clearly show the validity of Eq. (21).

On the other hand, it is interesting to explore the effect of the degree of orthotropy on the stress intensity factor. In particular, in what follows we consider the effect of high orthotropy. This was accomplished by considering large differences in the orthotropic constants in the directions parallel and perpendicular to the crack line. The material behavior of plywood was utilized.

In the first case the strong direction coincides with the crack axis: $E_{1}=24.600$, $E_{2}=1.000, v_{1}=0.298, v_{2}=0.012$ and $G=0.750$. Equation (21) then gives $K_{I}^{(\alpha)}=1.346$ $u_{0} h^{-1 / 2}$. In the second case the weak direction coincides with the crack axis: $E_{1}=1.000$, $E_{2}=24.600, v_{1}=0.012, v_{2}=0.298$ and $G=0.750$. In that case Eq. (21) gives $K_{I}^{(b)}=30.549 u_{0} h^{-1 / 2}$. This great difference in SIF values for case $(\alpha)$ and $(b)$ is mainly due to the term $c_{22}$ in the numerator of Eq. (21).

\section{References}

[1] M. F. Kanninen and C. H. Popelar, Advanced fracture mechanics. Oxford University Press, New York 1985.

[2] H. G. Georgiadis and G. A. Papadopoulos, Determination of SIF in a cracked plane orthotropic strip by the Wiener-Hopf technique. Int. J. Fracture 34, 57-64 (1987).

[3] W. G. Knauss, Stresses in an infinite strip containing a semi-infinite crack. J. Appl. Mech. 33, 356-362 (1966).

[4] J. R. Rice, Discussion on Ref. [3]. J. Appl. Mech. 34, 248-249 (1967).

[5] F. Nilsson, Dynamic stress-intensity factors for finite strip problems. Int. J. Fracture 8, 403-411 (1972).

[6] C. H. Popelar and C. Atkinson, Dynamic crack propagation in a viscoelastic strip. J. Mech. Phys. Solids 28, 79-93 (1980).

[7] B. R. Baker, Ductile yielding and brittle fracture at the ends of parallel cracks in a stretched orthotropic sheet. Int. J. Fracture 2, 576-595 (1966).

[8] Y. Konishi and A. Atsumi, Crack problem of transversely isotropic strip. Int. J. Engng. Sci. 11, 9-20 (1973).

[9] P. K. Satapathy and H. Parhi, Stresses in an orthotropic strip containing a Griffith crack. Int. J. Engng. Sci. 16, 147-154 (1978).

[10] G. C. Sih and E.P. Chen, Cracks in composite materials, Mechanics of Fracture 6. Nijhoff Publ., The Hague 1981.

[11] A. Cinar and F. Erdogan, The crack and wedging problem for an orthotropic strip. Int. J. Fracture 26, 83-102 (1983).

[12] S. G. Lekhnitskii, Theory of elasticity of an anisotropic elastic body. Holden-Day, San Francisco 1963.

[13] G. F. D. Duff and D. Naylor, Differential equations of applied mathematics. Wiley, New York 1966. 
[14] B. Noble, Methods based on the Wiener-Hopf technique. Pergamon Press, New York 1958.

[15] H. G. Georgiadis, Complex-variable and integral-transform methods for elastodynamic solutions of cracked orthotropic strips. Engng. Fracture Mech. 24, 727-735 (1986).

\section{Abstract}

The stress intensity factor at the tip of a semi-infinite crack in an orthotropic infinite strip was determined. Clamped strip boundaries were considered.

(Received: February 21, 1987; revised: December 2, 1987) 